\title{
Feedback Nash Equilibrium of N Agents Fishery Problem
}

\author{
Letian $\mathrm{Jiao}^{1} \&$ Haitao Chen $^{2}$ \\ ${ }^{1}$ China Economics and Management Academy, Central University of Finance and Economics, Beijing, China \\ ${ }^{2}$ School Of Economics, Central University of Finance and Economics, Beijing, China \\ Correspondence: Haitao Chen, School Of Economics Central University of Finance and Economics, Beijing, \\ China.
}

Received: October 21, 2020

Accepted: December 7, 2020

Online Published: April 26, 2021

doi:10.5539/ibr.v14n5p78

URL: https://doi.org/10.5539/ibr.v14n5p78

\begin{abstract}
This paper is built on the fundamental of Jorgensen and Sorge considering a differential game about fishery problem. In reality, the exploiters can be many because of the non-excludability of common resource. Thus, we expand the former two players model to $\mathrm{N}$ players model and we find more different equilibriums in $\mathrm{N}$ players scenario. Through this, we want to find some guidance for the changing of common resource stock. Further to control overexploitation.
\end{abstract}

Keywords: feedback Nash equilibrium, fishery, resource, extinction

\section{Introduction}

As economy develops, resource exploitation problem is being more and more important. As common public good, the negative externality of nature resource exploitation is always bothering us. For example, over fishery in ocean or lake, over exploitation of mineral, and forest overcutting. Fishing rights is still a severe conflict in international country. Because of the externality of exploitation, the resource is always in an inefficient state. To make good use of nature resource and control the exploitation behavior, governments, all kinds of international organizations have published regulation about the exploitation of nature resource such that closed fishing season. In order to manage the nature resource and keep it at an efficient renew level, we must study the exploiters' behavior given resource renewing discipline. For nature resource, it has some power to recover themselves. The recovery rate of nature resource is non-linear. When the stock of nature resource is low, the recovery rate is low because the recovering is restrained by the number of nature resource. As the stock of nature resource increases, the recovery rate increases. However, when the stock of nature resource exceeds some level the limited environment becomes a new bind, the recovery rate decreases as the stock of nature resource keeps increasing. According to this nature resource recovering principle, it is best to keep the stock of nature resource at the level of fast recovering rate. But this outcome may not be attainable with decentralized strategy that everyone only considers their own profit.

In our paper, we consider a differential games model of fishery trying to predict the exploiters behavior and the equilibrium of fishery stock. We analyze both infinite-horizon games and finite-horizon games respectively and allow exploiters to be heterogenous. And we consider who is going to exploit with exploiters being different in efficiency. The purpose of the model is to study overexploitation and environment degeneration in order to predict the future consequence.

We organize the paper as follows. Section 2 sets the model of fishery differential games. Section 3 exhibits some concept of feedback Nash equilibrium. Section 4 and section 5 are the most important part which we extend the Ref.1. Conclusion is given in section 7. The proof of our proposition is in the appendix.

In section 4 , we consider a different equilibrium of infinite-horizon game. In the research by Jorgensen and Sorger (1990), authors only consider the equilibrium that the bionomic equilibrium stock is at the level of the less efficient agent, but we consider a more natural equilibrium. Because at that stock level, the less efficient agent will never exploit the resource. There is only one agent who is the most efficient remain exploiting. Therefore, we constitute an equilibrium that the differential game degenerate to an optimal control problem in section 4, and the other $N-1$ agents have no incentive to join the game. And we consider a similar equilibrium of extinction to Jorgensen and Sorger (1990). We also correct a mistake of $G(x)$ in Jorgensen and Sorger (1990) and constitute a playable feedback Nash equilibrium under some similar assumption. 
In section 5, we consider finite-horizon game. In a $N$ agents game, which is different from 2 agents game, there is only one feedback Nash equilibrium that no one exploit before the game ends. This is important that we could use this result to protect a common-resource and make it achieve its maximal payoff. We also make a sensitive analysis to predict the influence of the change of agents' preference.

\section{Literature Review}

Research by Jorgensen and Sorger (1990) studied two players exploiting fish resource, they considered Nash equilibrium of biological equilibrium and extinction in scenario of both infinite horizon and finite horizon. However, there may be more potential players in the game without actions waiting to exploit in reality. If the stock of fish is high enough, there will be positive profit to make for many players. In this paper, we extend the exhaustible fishery differential game model of Jorgensen and Sorger (1990) to $N$ agents' fishery game. We build their feedback Nash equilibrium strategies in order to predict the exploiting efforts of every player in different resource stock level. In infinite horizon game, Jorgensen and Sorger (1990) only build an equilibrium of keeping the equilibrium stock at the level of excluding other players. But if there is large difference between the efficiency of players, only the most efficient players can make profit in a level of stock much lower than other players' preferred level, there is no need for this exploiting leader to keep other players out but exploit at the level he favors. A relative low level of stock will be preferred by this player. In this scenario, the equilibrium resource stock may be lower than before. Therefore, in common resource management, we should keep our eyes on the leader exploiter. This exploiter's efficient level is important, deciding the equilibrium level. Intuitively, the standard of an industry with competitiveness is highly influenced by the leader of this industry. Every competitor is trying to approach this standard. This standard also signals the limitation of the industry now. So, it is rational that the leader's efficiency level highly influences the equilibrium level. If this level is too low than a government preferred level, we can improve the equilibrium stock by increasing the efficient level of the leader. In two different scenarios mentioned above, the outcomes can be vastly distinct. When the equilibrium is for leader to exclude competitor, we can change the equilibrium by influencing the efficiency of other exploiters. But if the equilibrium is the solution of leader's own optimal control problem, we can only change the equilibrium by influencing the leader. In finite horizon game, we find that only no exploiting and waiting to harvest is the best choice. And we present a sensitive analysis to study that what factors changing can make players deviate from waiting for harvest equilibrium. We also consider what factors influence the value of players. Research by Jorgensen (1985) studies an exponential differential game, and presents an optimal exploitation of exhaustible resource. He proves the "equilibrium in open-loop controls is also close-loop equilibrium" in fishery game, the choices of players are of no time-inconsistency. Lewis (1981) studies a discontinuous exploitation model, considers the resource renewing process is influenced by some stochastic factors and find a close-loop strategy to maximize discounted expectation social return. Mundy and Mathiesen (1981) estimate the economic benefit from managing fishery by measuring the weight change of salmon fisheries accurately. Dockner, Jorgensen, Long, and Sorger (2000) summarize the methodology broadly uses in differential games. Levhari and Mirman (1980) consider fishery game in the frame of Cournot-Nash equilibrium and study how players influence each other in the game. Player' equilibrium strategy depends on others' strategies and the stock of fish but not on all players' previous behavior. Research by Clemhout and Wan analyze many species of fish and the interaction between species. They also consider the stochasticity of nature. Clark (1980) studies property of fishery and the influence by restricting entrance on players and environment. Research by Janssen, Tyson and Lee (2014) support that the dilemma of common resource can be improved and corporation between players can be increased if exploiters can communicate with each other at some cost. Under more corporation by communication, group performance is better than decentralized strategy. Karpoff (1987) studies the influence of season closure and capital constraints on players' strategies, which say the regulation leads to redistribution from efficient fishermen to inefficient fishermen. These regulations cannot help resource state to be optimal and it can only improve the performance of players in some extent.

\section{Model Set}

\subsection{State Equation}

In our common-property fishery resource exploitation problem, we suppose that there are finitely many agents who make their decision independently. They are exploiting a same kind of fish in fish resource stock. Let $x=x(t)$ denote the fish stock of this species that can be exploit at time $t, 0 \leq t \leq T$, where $T$ denotes the length of time horizon of making decision. Time horizon $T$ can be fixed and either finite or infinite. We assume the growth rate of the fish resource stock follows logistic, that is, 


$$
\mathrm{y}(\mathrm{x})=\mathrm{rx}\left(1-\frac{x}{k}\right)
$$

where $\mathrm{g}$ is the natural growth function of fishery resource stock, $r=$ constant $>0$ is the natural growth rate of fish, and $k=$ constant $>0$ denotes the carrying ability of the environment.

Let $u_{t}(t)$ be the exploit effort, We assume that the total harvest rate is

$$
h\left(u_{1}, \cdots, u_{N}, x\right)=b x\left(e^{\sum_{i=1}^{N} u_{i}}-1\right)
$$

where $b=$ constant $>0$ denotes the exploit effect.

Combine (1) and (2) we get state equation describing the change of fish resource stock,

$$
\dot{x}=r x\left(1-\frac{x}{k}\right)-b x\left(e^{\sum_{i=1}^{N} u_{i}}-1\right)
$$

\subsection{Payoff Function}

Suppose that investor $k$ 's payoff function is

$$
J^{k}=\int_{0}^{T} c_{k} x e^{-p_{k} t} e^{u_{k}+a_{k} \sum_{i \neq k} u_{i}} d t+e^{-p_{k} T} S_{k} x(T) .
$$

where $k \in\left\{\mathrm{i} \in \mathrm{N}^{+}: 1 \leq i \leq N\right\}$.

In (4), $c_{k}$ is a positive constant demonstrating the payoff gained by agent $k, a_{k}$ is constant demonstrating the sensitivity of payoff to other agents' exploit efforts, and $S_{i}$ is not less than zero and constant demonstrating the salvage value per fish stock remained when the game is over. In the infinite horizon case $T=\infty$, we let $S_{i}=0$. And the discount factor $p_{k}$ is a positive constant.

\section{Definition of Feedback Nash Equilibrium}

With an assumption of feedback information structure, we suppose that each agent knows the fishery resource stock at each decision time, and agents' strategies depend on the current fish resource stock $x$ and time $t$. We make assumption of information structure according to Lewis(1981) and Mundy and Mathiesen(1981). For a feedback strategy has advantages of time consistency and subgame perfectness compared to the open-loop strategy according to Dockner, Jorgensen, Long \& Sorger(2000). Feedback solution of similar problem have been analyzed for example, in Levhari and Mirman(1980), Clemhout, and Wan(1985).

The state variable of fish stock $x(t)$ must not be outside of the interval $[0, k]$,for all $t \in[0, T]$.Because the fish stock cannot be negative. And natural fish growth function has a decreasing marginal effect so fish stock cannot be greater than some value $k$,which is a biggest capacity. The control $u_{i}(t)$ must satisfy $u_{i}(t) \in\left[0, u_{i}^{m}\right]$,for all $\mathrm{t}$, where $u_{i}^{m}=$ constant $>0$ means the maximal exploit efforts which can be made by investor $i$.

Definition 4.1. The feedback strategy for investor $i$ is a function $v_{i}$ from $[0, k] \times[0, T]$ to $U_{i}=\left[0, u_{i}^{m}\right]$.

Thus, $u_{i}(t)=v_{i}(x(t), t)$ is the corresponding open-loop strategy when strategy $v_{i}(x, t)$ is used.

Definition 4.2. A control path is $v_{i}(x, t)$ feasible if there is a unique absolutely continuous function $x(t)$ such that $x(t) \in[0, k]$ and $v_{i}(x, t) \in\left[0, u_{i}^{m}\right]$ hold for all $\mathrm{t}$ and (4) is well defined.

For the un-centralized $\mathrm{N}$-investor game with state equation

$$
\dot{x}=r x\left(1-\frac{x}{k}\right)-b x\left[e^{\sum_{i=1}^{N} u_{i}}-1\right], x(0)=x_{0}
$$

and payoff function defined by (4), we define the equilibrium of the game.

Definition 4.3. A strategy combination $\left\{v_{i}^{*}\right\}$ is a feedback Nash equilibrium if it is feasible and

$$
J^{i}\left(v_{1}^{*}, \cdots, v_{i}^{*}, \cdots, v_{N}^{*}\right) \geq J^{i}\left(v_{1}^{*}, \cdots, v_{i}, \cdots, v_{N}^{*}\right),
$$


for all playable $v_{i}$, for all $i \in\left\{\mathrm{i} \in \mathrm{N}^{+}: 1 \leq i \leq N\right\}$.

Given that other agents all use her feedback Nash equilibrium strategy, then investor $i$ will not deviate from her feedback Nash equilibrium strategy.

\section{Fishery Game of Infinite-Horizon}

Within this section, we consider the differential game defined by the state equation (3) and the payoffs function (4). This scenario is that there are $\mathrm{N}$ agents are expected to exploit in the long run. In section 5.1, we solve the game by introducing a description of positive fish stock equilibrium concept according to Clark(1980). Next, in section 5.2, we solve the extinction equilibrium.

\subsection{Solution with Positive Fish Stock}

Define $x_{i}^{\infty}$ as the lowest fish stock for agent $i$ to earn a positive profit. For any $\mathrm{x}<x_{i}^{\infty}$, the instant profit is negative, which implies the agent should not keep exploiting. Furthermore, we say that agent $i$ has an efficient advantage than agent $j$ if $x_{i}^{\infty}<x_{j}^{\infty}$;i.e, agent $i$ can earn a positive economic profit on a smaller fish resource stock level than agent $j$. There are two different feedback Nash equilibrium worth to notice. Therefore, we exhibit Proposition 5.1 and Proposition 5.2.

Proposition 5.1 Without losing of generality, we assume that the efficiency is increasing in order as 1 to $\mathrm{N}$. That is $x_{1}^{\infty}<x_{2}^{\infty}<\cdots<x_{N}^{\infty}$. Denote by $\tilde{x}_{i}=\frac{k\left(b+r-p_{i}\right)}{2 r}$ the sole-owner singular level. The strategies

$$
\begin{aligned}
& v_{1}^{*}(x, t)=\left\{\begin{array}{l}
u_{1}^{m}, x>\tilde{x}_{1} \\
u_{1}^{s}, x=\tilde{x}_{1} \\
0, x<\tilde{x}_{1}
\end{array}\right. \\
& v_{i}^{*}(x, t)=\left\{\begin{array}{c}
u_{i}^{m}, x>x_{i}^{\infty} \\
0, x \leq x_{i}^{\infty}
\end{array}, i=2,3, \cdots, N\right.
\end{aligned}
$$

where

$$
u_{1}^{s}=\ln \left[\frac{F(x)}{b x}\right]
$$

yield a feedback Nash equilibrium, if following conditions are satisfied:

$$
\begin{aligned}
& x_{1}^{\infty}<\tilde{x}_{1} \leq x_{2}^{\infty}<\tilde{x}_{2} \leq \cdots \leq x_{N}^{\infty}<\tilde{x}_{N}
\end{aligned}
$$

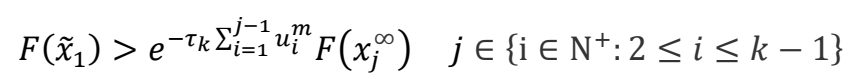

$$
\begin{aligned}
& F\left(\tilde{x}_{1}\right)>e^{-\tau_{k} \sum_{i \in\left\{i \in \mathrm{N}^{+}: 1 \leq i \leq j, i \neq k\right\}} u_{i}^{m}} F\left(x_{j}^{\infty}\right) j \in\left\{\mathrm{i} \in \mathrm{N}^{+}: k-1 \leq i \leq N\right\} \\
& F\left(\tilde{x}_{1}\right)>e^{-\tau_{k} \sum_{i=1}^{k-1} u_{i}^{m}} F\left(\tilde{x}_{k}\right)
\end{aligned}
$$

for $j \in\left\{\mathrm{i} \in \mathrm{N}^{+}: 1 \leq i \leq k-2\right\}$.

Remark 5.1. Assumption (7) satisfies if every agent has a sufficiently large maximal efforts choice. That is $u_{i}^{m}$ is sufficiently large.

Proposition 5.2 Without losing of generality, we assume that the efficiency is increasing in order as 1 to N. That is $x_{1}^{\infty}<x_{2}^{\infty}<\cdots<x_{N}^{\infty}$. Denote the sole-exploiter singular level given by $\frac{k(b+r-p)}{2 r}$, and replaced $p$ by $p_{i}$. The strategies

$$
\begin{aligned}
& v_{1}^{*}(x, t)=\left\{\begin{array}{c}
u_{1}^{m}, x_{2}^{\infty}<x \leq k \\
u_{1}^{s}, \quad x=x_{2}^{\infty} \\
0, \quad 0 \leq x<x_{2}^{\infty}
\end{array}\right. \\
& v_{i}^{*}(x, t)=\left\{\begin{array}{c}
u_{i}^{m}, x_{i}^{\infty}<x \leq k \\
0, x \leq x_{i}^{\infty}
\end{array}, i=2,3, \cdots, N\right.
\end{aligned}
$$


where $u_{1}^{s}$ is defined as (6), yield a feedback Nash equilibrium, if following conditions are satisfied:

$$
\begin{aligned}
& x_{j}^{\infty}<\tilde{x}_{1} \leq x_{j+1}^{\infty} \\
& F\left(x_{2}^{\infty}\right)<b x_{2}^{\infty} e^{u_{1}^{m}} \\
& F\left(x_{k}^{\infty}\right)>e^{-\tau_{1} u_{k}^{m}}\left[F\left(x_{k+1}^{\infty}\right)-p_{1}\left(x_{k+1}^{\infty}-x_{k}^{\infty}\right)\right] \\
& \text { for } k \in\left\{\mathrm{i} \in \mathrm{N}^{+}: 2 \leq i \leq j-1\right\} \\
& F\left(x_{2}^{\infty}\right)>e^{-\tau_{1} \Sigma_{2}^{j} u_{i}^{m}}\left[F\left(\tilde{x}_{1}\right)-p_{1}\left(\tilde{x}_{1}-x_{j}^{\infty}\right)\right]-p_{1}\left(x_{j}^{\infty}-x_{j-1}^{\infty}\right) e^{-\tau_{1} \sum_{2}^{j-1} u_{i}^{m}}-\cdot \cdot \\
& -p_{1}\left(x_{3}^{\infty}-x_{2}^{\infty}\right) e^{-\tau_{1} u_{2}^{m}} \\
& F\left(x_{j}^{\infty}\right)>e^{-\tau_{k} I_{j j \neq k\}} u_{j}^{m}}\left[F\left(x_{j+1}^{\infty}\right)-p_{k}\left(x_{j}^{\infty}-x_{j-1}^{\infty}\right)\right],
\end{aligned}
$$

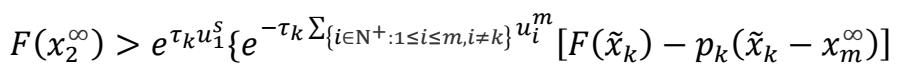

$$
\begin{aligned}
& -p_{k}\left(x_{m}^{\infty}-x_{m-1}^{\infty}\right) e^{-\tau_{k} \sum_{i \in\left\{i \in \mathrm{N}^{+}: 1 \leq i \leq N-1, i \neq k\right\}} u_{i}^{m}} \\
& \left.-\cdots-p_{k}\left(x_{3}^{\infty}-x_{2}^{\infty}\right) e^{-\tau_{k}\left(u_{1}^{m}+u_{2}^{m}\right)}\right\}
\end{aligned}
$$

for some $j \in\left\{\mathrm{i} \in \mathrm{N}^{+}: 2 \leq i \leq m-1, i \neq k-1, i \neq k\right\}, \forall m \in\left\{\mathrm{i} \in \mathrm{N}^{+}: 2 \leq i \leq N-1\right\}$.

$\forall k \in\left\{\mathrm{i} \in \mathrm{N}^{+}: 2 \leq i \leq N\right\}$

The difference between Proposition 5.1 and Proposition 5.2 depends on the difference between the efficiency of player 1 and player 2. If the efficiency of player 1 is much higher than player 2's, we may predict an equilibrium of Proposition 5.1. The player 1 just choose his optimal control option. If the efficiency of player 1 is close to player 2's, we may predict an equilibrium of Proposition 5.2. The player 1 keep the fish stock at the level of excluding player 2.

\subsection{Solution to Extinction Equilibrium}

In following, we analyze an equilibrium of the game which is not in bionomic equilibrium. Then exploit will be like a race.

Proposition 5.3 The strategies

$$
v_{i}(x)=\left\{\begin{array}{l}
u_{i}^{0}, x=0 \\
u_{i}^{m}, x>0
\end{array}, i \in\left\{\mathrm{i} \in \mathrm{N}^{+}: 1 \leq i \leq N\right\}\right.
$$

constitute a feedback Nash equilibrium if the following inequality satisfies

$$
r+b-p_{i} \leq 0 .
$$

The values $u_{i}^{0}$ is a constant such that $u_{i}^{0} \in\left[0, u_{i}^{m}\right]$.

Remark 5.2. The arbitrariness of $u_{i}^{0}$ is irrelevant, because no matter what value $u_{i}^{0}$ are $J^{i}$ is unchangeable after $x$ gets to zero, whatever strategy pair $\left\{v_{i}(x)\right\}, i \in\left\{\mathrm{i} \in \mathrm{N}^{+}: 1 \leq i \leq N\right\}$ agents choose. The value of $u_{i}^{0}$ has no influence, and any admissible $u_{i} \in\left[0, u_{i}^{m}\right]$ are satisfied.

Proposition 5.2 explain that agents choose maximal effort as long as there is a positive resource stock. This makes sense with economic intuition because the assumption (17) means that the discount rate is large because of great risk etc. Agents are trying to escape from the capital market of this country.

Next, consider the case of no great risk to increase the discount rate, and we suppose that 


$$
r+b-p_{i}>0
$$

We define the constants

$$
\tilde{x}_{u}=\max \left\{\tilde{x}_{i}\right\}, \tilde{x}_{d}=\min \left\{\tilde{x}_{i}\right\}, i \in\left\{\mathrm{i} \in \mathrm{N}^{+}: 1 \leq i \leq N\right\},
$$

the functions

$$
G_{j}(x)=\frac{\ln \left[x^{r+b-p_{j}} \cdot\left(r+b-\frac{r x}{k}\right)^{r+b+p_{j}}\right]}{\tau_{j}(r+b)},
$$

and the singular control

$$
u^{s}=\ln \left[\frac{F(x)}{b x}\right]
$$

The monotonicity of $G_{j}(x)$ is same as $h(x)=x^{r+b-p_{j}} \cdot\left(r+b-\frac{r x}{k}\right)^{r+b+p_{j}}$.

$$
\begin{aligned}
& h_{j}^{\prime}(x)=\left(r+b-p_{j}\right) x^{r+b-p_{j}-1}\left(r+b-\frac{r x}{k}\right)^{r+b+p_{j}}+\left(r+b+p_{j}\right) x^{r+b-p_{j}} \cdot\left(r+b-\frac{r x}{k}\right)^{r+b+p_{j}-1} \\
& \quad=x^{r+b-p_{j}-1}\left(r+b-\frac{r x}{k}\right)^{r+b+p_{j}-1}\left[\left(r+b-p_{j}\right)\left(r+b-\frac{r x}{k}\right)+\left(r+b+p_{j}\right) x\right] \\
& \quad>0
\end{aligned}
$$

Thus $G_{j}(x)$ is increasing for $x \in[0, k]$.

Lemma 5.1. Suppose that (i) $u_{k}^{m}>u^{s}$ (ii) $\frac{\sum_{1}^{N} G_{i}\left(\tilde{x}_{u}\right)}{N-1}-G_{k}\left(\tilde{x}_{d}\right)<u_{k}^{m}$ and (iii) $\frac{\sum_{1}^{N} G_{i}(x)}{N-1}-G_{k}(x)>0$ for $k \in$ $\left\{\mathrm{i} \in \mathrm{N}^{+}: 1 \leq i \leq N\right\}$.Then, there are infinite pairs $(\underline{x}, \bar{x})$ satisfying following conditions for $i \in\left\{\mathrm{i} \in \mathrm{N}^{+}: 1 \leq\right.$ $i \leq N\}$ :

$$
\begin{aligned}
& 0<\underline{x} \leq \tilde{x}_{d} \leq \tilde{x}_{u} \leq \bar{x} \leq k, \\
& \frac{\sum_{1}^{N} G_{i}(\bar{x})}{N-1}-G_{k}(\underline{x}) \leq u_{k}^{m}, \\
& \sum_{k=1}^{N}\left[\frac{\sum_{1}^{N} G_{i}(\bar{x})}{N-1}-G_{k}(\underline{x})\right] \geq u^{s} .
\end{aligned}
$$

Proof. We make $\bar{x}=\tilde{x}_{u}$ and show that there are infinite $\tilde{x}_{d}$ satisfying (22). The function $G_{j}(x)$ is strictly increasing in $x \in[0, k], G_{j}(x) \rightarrow-\infty$ when $x \rightarrow 0$. By assumption (ii) and the monotonicity of function $G_{j}(x)$, we can get that given fixed $\bar{x}$, the existence of a unique value $z_{j} \in\left(0, \tilde{x}_{d}\right)$ such that $\frac{\sum_{1}^{N} G_{i}(\bar{x})}{N-1}-G_{k}\left(z_{k}\right)=$ $u_{i}^{m}, \forall \mathrm{k} \in\left\{\mathrm{i} \in \mathrm{N}^{+}: 1 \leq i \leq N\right\}$.Therefore,

$$
\tilde{z}=\max \left\{z_{i}\right\}, i \in\left\{\mathrm{i} \in \mathrm{N}^{+}: 1 \leq i \leq N\right\}<\tilde{x}_{d} .
$$

According to the definition of $\tilde{z}$, we have

$$
0<\frac{\sum_{1}^{N} G_{i}(\bar{x})}{N-1}-G_{k}(\tilde{z}) \leq u_{i}^{m}, \mathrm{j} \in\left\{\mathrm{i} \in \mathrm{N}^{+}: 1 \leq i \leq N\right\} . .
$$

And we have 


$$
\begin{aligned}
& \sum_{k=1}^{N}\left[\frac{\sum_{1}^{N} G_{i}(\bar{x})}{N-1}-G_{k}(\tilde{z})\right]=u_{j}^{m}+\sum_{k \neq j}\left[\frac{\sum_{1}^{N} G_{i}(\bar{x})}{N-1}-G_{k}(\tilde{z})\right] \\
& >u_{j}^{m}+\sum_{k \neq j}\left[\frac{\sum_{1}^{N} G_{i}(\bar{x})}{N-1}-G_{k}(\bar{x})\right] \\
& >u_{j}^{m} \\
& >u^{s} .
\end{aligned}
$$

By continuity, there exists a constant $\theta$ satisfying conditions (19) for all $\underline{x} \in[\tilde{z}, \tilde{z}+\theta]$.

Till now, we have only constructed pairs $(\underline{x}, \bar{x})$ with $\bar{x}=\tilde{x}_{u}$. But by continuity, pairs with $\bar{x}>\tilde{x}_{u}$ and satisfying (19) also exist.

Proposition 5.4. Given that (17) is satisfied and that assumption (i)-(iii) of Lemma 5.1 are satisfied. Then the following strategies form a feedback Nash equilibrium:

$$
\begin{aligned}
& v_{k}(x) \\
& =\left\{\begin{array}{cc}
0, & 0 \leq x \leq \underline{x} \\
{\left[\frac{\sum_{1}^{N} G_{i}(x)}{N-1}-G_{k}(x)\right]-\left[\frac{\sum_{1}^{N} G_{i}(\underline{x})}{N-1}-G_{k}(\underline{x})\right],} & \underline{x} \leq x<\bar{x} \\
\bar{u}_{k}, & x=\bar{x} \\
u_{i}^{m}, & \bar{x}<x \leq k
\end{array}\right.
\end{aligned}
$$

where $k \in\left\{\mathrm{i} \in \mathrm{N}^{+}: 1 \leq i \leq N\right\}$.The constants $\bar{u}_{k} \in\left[\frac{\sum_{1}^{N} G_{i}(\bar{x})}{N-1}-G_{k}(\underline{x}), u_{i}^{m}\right]$ are arbitrary.

In $(19),(\underline{x}, \bar{x})$ is an arbitrary pair, Lemma 5.1 guarantees its existence.

Remark 5.3. The idea of the constructing strategies (19) is to extend the switching point of Proposition 5.1 and Proposition 5.2 into a nonvoid singular interval $[\underline{x}, \bar{x})$, which is necessary because the strategies with only one switching point are not playable in reality. We can overcome these difficulties in Proposition 5.1 and Proposition 5.2 by introducing the capital equilibrium stock.

Finally, using the strategies in (19) causes the state path to converge to a positive fish resource stock equilibrium level, which will be determined by constant $\bar{x}$ and $\underline{x}$.

\section{Fishery Game of Finite-Horizon with Salvage Value}

In this section, we consider the differential game with evolution equation (3) and payoffs function given by (4). Fishery will close at some date that can be known by agents. And every agent can know their salvage value $S_{i}$. In this scenario, differential game will end at some $T$. This section presents equilibrium that every agent does not exploit and wait for the end of game.

Proposition 6.1. Then, the strategies $v_{i}(t, x)=0$ constitute the only feedback Nash equilibrium, and the value function of agent $i$ is

$$
V^{k}(t, x)=\frac{\pi_{i} e^{-p_{k}} S_{k}(r+b)}{\frac{r}{k}+\left(\frac{r+b}{x}-\frac{r}{k}\right) e^{(r+b)(t-T)}} .
$$

where $\pi_{i}$ is a constant demonstrating the profitable ability of agent $i$. 
Remark 6.1. The preference of agent whose payoff is more sensitive to other agent's efforts $\left(a_{i}\right.$ is closer to 1$)$ must value today's payoff less ( $c_{i}$ is small) or value salvage value more $\left(S_{i}\right.$ is large) or have low discount rate $\left(p_{i}\right.$ is small) to keep the equilibrium.

The agent $k$ will be more willing to do something if he is less sensitive to other agents' $\operatorname{effect}\left(a_{k}\right.$ is closer to 0$)$ or he values salvage value more $\left(S_{k}\right.$ is large $)$ or he values today's payoff less $\left(c_{k}\right.$ is small) or he has a small discount rate $\left(p_{k}\right.$ is small).

\section{Conclusion}

In this paper, we has dealt with the problem of $\mathrm{N}$ agents exploiting the fish resources without cooperation. We constitute two bionomic equilibrium, an extinction equilibrium and a playable equilibrium in an interval in infinite-horizon game. And we constitute a no-exploit equilibrium in finite-horizon game.

When there is an agent whose efficiency is high, the game may become its own optimal control problem rather than keeping stock at some higher level to avoid other agents to join. This scenario happens when the efficiency of the most efficient agent is much higher than others. In this scenario, the leader of exploiters is a key player. If the resource stock of equilibrium is too low for the society, we should focus on the efficient level of the leader rather than influence every other player.

In finite-horizon scenario, when agents increasing from 2 to $N(N>2)$, there is only one feedback Nash equilibrium which is every agent stop exploiting and wait for the end of the game to get the salvage value. And the incentive to deviate from this equilibrium has positive relationship with unit salvage value $\left(S_{k}\right)$ and negative relationship with profitable ability $\left(c_{k}\right)$, sensitivity to others' efforts $\left(a_{k}\right)$ and discount $\operatorname{rate}\left(p_{k}\right)$, which is also intuitive.

\section{References}

Clark, C. W. (1980). Restricted access to common-property fishery resources: A game theoretic analysis, Dynamic Optimization and Mathematical Economics. Edited by P. T. Liu, Plenum Press, New York, 117-132. https://doi.org/10.1007/978-1-4684-3572-6_7

Clemhout, S., \& Wan, H. Y. (1985). Dynamic common property resources and environmental problems. Journal of Optimization Theory and Applications, 46(4), 471-481. https://doi.org/10.1007/BF00939152

Dockner, E. J., Jorgensen S., Long, N. V., \& Sorger, G. (2000). Differential games in economics and management science (1st ed.). Cambridge University Press. https://doi.org/10.1017/CBO9780511805127

Janssen, M., Tyson, M., \& Lee, A. (2014). The effect of constrained communication and limited information in governing a common resource. International Journal of the Commons, 8(2), 617-635. https://doi.org/10.18352/ijc.473

Jorgensen, S. (1985). An exponential differential game which admits a simple Nash solution. Journal of Optimization Theory and Applications, 45(3), 383-396. https://doi.org/10.1007/BF00938442

Jorgensen, S., \& Sorger, G. (1990). Feedback Nash equilibrium in a problem of optimal fishery management. Journal of Optimization Theory and Applications, 64(2). https://doi.org/10.1007/BF00939450

Karpoff, J. M. (1987). Suboptimal controls in common resource management: The case of the fishery. Journal of Political Economy, 95(1), 179-194. https://doi.org/10.1086/261447

Levhari, D., \& Mirman, L. J. (1980). The great fish war: An example using a dynamic Nash-Cournot solution. Bell Journal of Economics, 11(1), 322-334. https://doi.org/10.2307/3003416

Lewis, T. R. (1981). Exploitation of a renewable resource under uncertainty. Canadian Journal of Economics, 14(3), 422-439. https://doi.org/10.2307/134897

Mundy, P. R., \& Mathiesen, O. A. (1981). Abundance estimation in a feedback control system applied to the management of a commercial salmon fishery. Applied operations research in fishing. Edited by K. B. Haley, Plenum Press, New York, New York. https://doi.org/10.1007/978-1-4613-3222-0_6 


\section{Appendix}

Proof of Proposition 5.1

The state equation (3) yields

$$
x e^{\sum_{1}^{N} u_{i}}=\frac{F(x)-\dot{x}}{b} .
$$

Substitute (A1) into the payoffs function $J^{k}$ given by (4) and integrate it by parts

$$
\begin{gathered}
J^{k}=\int_{0}^{\infty} c_{k} \cdot \frac{F(x)-\dot{x}}{b} \cdot e^{-\sum_{1}^{N} u_{i}(x)} \cdot e^{-p_{k} t} \cdot e^{u_{k}+a_{k} \sum_{i \neq k} u_{i}} d t \\
=\frac{c_{k}}{b} \int_{0}^{\infty}[F(x)-\dot{x}] e^{-p_{k} t} \cdot e^{-\sum_{i \neq k} \tau_{k} u_{i}(x)} d t \\
=\frac{c_{k}}{b}\left[\int_{0}^{\infty} F(x) e^{-p_{k} t} \cdot e^{-\sum_{i \neq k} \tau_{k} u_{i}(x)} d t-\int_{0}^{\infty} e^{-p_{k} t} \cdot e^{-\sum_{i \neq k} \tau_{k} u_{i}(x)} d x\right] \\
=\frac{c_{k}}{b}\left[\int_{0}^{\infty} F(x) e^{-p_{k} t} \cdot e^{-\sum_{i \neq k} \tau_{k} u_{i}(x)} d t-\int_{0}^{\infty} e^{-p_{k} t} d \int_{0}^{x} e^{-\sum_{i \neq k} \tau_{k} u_{i}(z)}\right] \\
=\frac{c_{k}}{b}\left[\int_{0}^{\infty} F(x) e^{-p_{k} t} \cdot e^{-\sum_{i \neq k} \tau_{k} u_{i}(x)} d t-\left.\left(e^{-p_{k} t} \int_{x_{0}}^{x} e^{-\sum_{i \neq k} \tau_{k} u_{i}(z)} d z\right)\right|_{0} ^{\infty}\right. \\
=\frac{c_{k}}{b} \int_{0}^{\infty} e^{-p_{k} t}\left[F(x) e^{-\sum_{i \neq k} \tau_{k} u_{i}(x)}-p_{k} \int_{0}^{x} e^{-\sum_{i \neq k} \tau_{k} u_{i}(z)} d z\right] d t+\text { constant } \\
=\frac{c_{k}}{b} \int_{0}^{\infty} e^{-p_{k} t} W_{i}(x) d t+\operatorname{costant}
\end{gathered}
$$

where

$$
\begin{gathered}
W_{k}(x)=F(x) e^{-\sum_{i \neq k} \tau_{k} u_{i}(x)}-p_{k} \int_{0}^{x} e^{-\sum_{i \neq k} \tau_{k} u_{i}(z)} d z . \\
\tau_{k}=1-a_{i}, k \in\left\{\mathrm{i} \in \mathrm{N}^{+}: 1 \leq i \leq N\right\}
\end{gathered}
$$

Agent k's optimal response to other agents' strategies $v_{i}(x), i \in\left\{\mathrm{i} \in \mathrm{N}^{+}: 1 \leq i \leq N, i \neq k\right\}$ is to approach to a level of $x$ as fast as possible which maximizes $W_{k}(x)$ subjects to $x \geq x_{k}^{\infty}$.

Suppose that agent $i, i \in\left\{\mathrm{i} \in \mathrm{N}^{+}: 2 \leq i \leq N\right\}$ use the strategies in (5). Then we have

$$
W_{1}(x)=\left\{\begin{array}{lr}
F(x)-p_{1} x, & 0 \leq x \leq x_{2}^{\infty} \\
e^{-\tau_{1} u_{2}^{m}\left[F(x)-p_{1}\left(x-x_{2}^{\infty}\right)\right]-p_{1} x_{2}^{\infty},} & x_{2}^{\infty}<x \leq x_{3}^{\infty} \\
e^{-\tau_{1}\left(u_{2}^{m}+u_{3}^{m}\right)}\left[F(x)-p_{1}\left(x-x_{3}^{\infty}\right)\right]-p_{1}\left(x_{3}^{\infty}-x_{2}^{\infty}\right) e^{-\tau_{1} u_{2}^{m}}-p_{1} x_{2}^{\infty}, & x_{3}^{\infty}<x \leq x_{4}^{\infty} \\
\cdots & \\
e^{-\tau_{1} \sum_{2}^{N} u_{i}^{m}}\left[F(x)-p_{1}\left(x-x_{N}^{\infty}\right)\right]-p_{1}\left(x_{N}^{\infty}-x_{N-1}^{\infty}\right) e^{-\tau_{1} \sum_{2}^{N-1} u_{i}^{m} \cdots-p_{1} x_{2}^{\infty}, x_{N}^{\infty}<x \leq k}
\end{array}\right.
$$

By assumption $x_{1}^{\infty}<\tilde{x}_{1}<x_{2}^{\infty}$,we know the maximum of $W_{1}(x)$ is attained at $x=\tilde{x}_{1}$ when $x$ is in the interval $\left[0, x_{2}^{\infty}\right]$, the problem degenerate to the optimal control problem studied before. And $W_{1}(x)$ decreases 
in each interval $\left(x_{i}^{\infty}, x_{i+1}^{\infty}\right], i \in\left\{\mathrm{i} \in \mathrm{N}^{+}: 1 \leq i \leq N-1\right\}$ and $\left(x_{N}^{\infty}, k\right)$ because $\tilde{x}_{1}$ satisfies

$$
\frac{d\left(F(x)-p_{1} x\right)}{d x}=0 \text { at } x=\tilde{x}_{1}
$$

so, when $x>\tilde{x}_{1}$

$$
\frac{d\left(F(x)-p_{1} x\right)}{d x}<0
$$

Thus, the supremum of each interval $\left(x_{i}^{\infty}, x_{i+1}^{\infty}\right], i \in\left\{\mathrm{i} \in \mathrm{N}^{+}: 1 \leq i \leq N-1\right\}$ and $\left(x_{N}^{\infty}, k\right)$ are their left limitation.

$$
\begin{aligned}
& W_{1}\left(x_{2}^{\infty}\right)-\lim _{x \rightarrow x_{2}^{\infty+}} W_{1}(x)=F\left(x_{2}^{\infty}\right)-p_{1} x_{2}^{\infty}-\left[e^{-\tau_{1} u_{2}^{m}} F\left(x_{2}^{\infty}\right)-p_{1} x_{2}^{\infty}\right] \\
& =\left(1-e^{-\tau_{1} u_{2}^{m}}\right) F\left(x_{2}^{\infty}\right)>0 \\
& W_{1}\left(x_{k}^{\infty}\right)-\lim _{x \rightarrow x_{k}^{\infty+}} W_{1}(x)=e^{-\tau_{1} \sum_{2}^{k-1} u_{i}^{m}\left[F\left(x_{k}^{\infty}\right)-p_{1}\left(x_{k}^{\infty}-x_{k-1}^{\infty}\right)\right]} \\
& -p_{1}\left(x_{k-1}^{\infty}-x_{k-2}^{\infty}\right) e^{-\tau_{1} \Sigma_{2}^{k-2} u_{i}^{m}}-\cdots-p_{1} x_{2}^{\infty}
\end{aligned}
$$

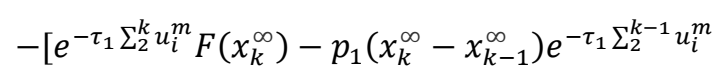

$$
\begin{aligned}
& \left.-p_{1}\left(x_{k-1}^{\infty}-x_{k-2}^{\infty}\right) e^{-\tau_{1} \sum_{2}^{k-2} u_{i}^{m}}-\cdots--p_{1} x_{2}^{\infty}\right] \\
& =\left(e^{-\tau_{1} \sum_{2}^{k-1} u_{i}^{m}}-e^{-\tau_{1} \sum_{2}^{k} u_{i}^{m}}\right) F\left(x_{k}^{\infty}\right)>0
\end{aligned}
$$

Therefore, the maximum of $W_{1}(x)$ is attained in the interval $\left[0, x_{2}^{\infty}\right]$. To be further, at $x=\tilde{x}_{1}$.

Now, suppose that agent $i, i \in\left\{\mathrm{i} \in \mathrm{N}^{+}: 1 \leq i \leq N, i \neq k\right\}$ use $v_{i}^{*}(x, t)$ given by (5).Then,

$$
\begin{aligned}
& W_{k}(x)
\end{aligned}
$$

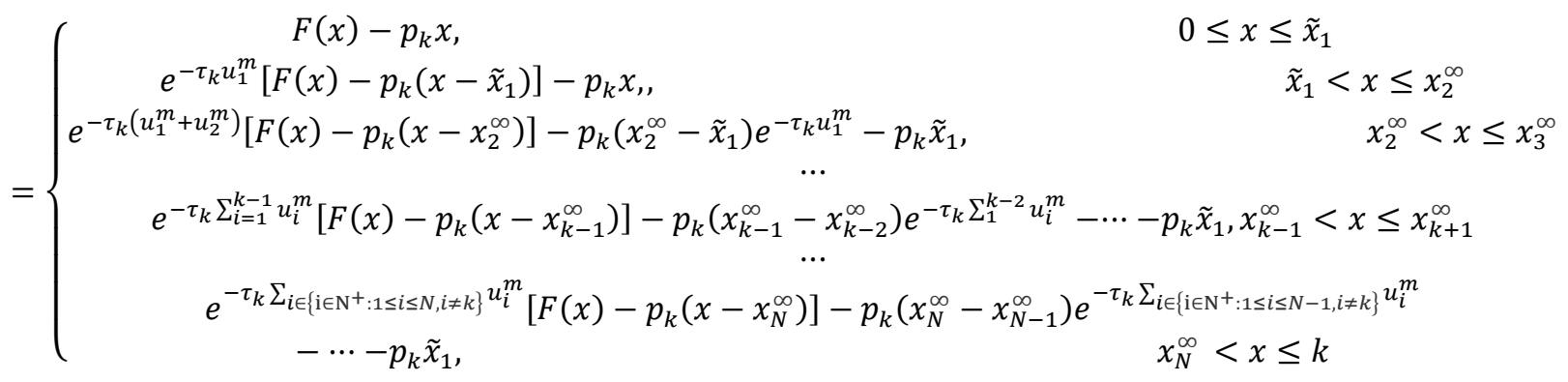

We want to show that $W_{k}(x)$ gets to its maximum at $x=\tilde{x}_{1}$,

$W_{k}(x)$ gets its maximum at $x=\tilde{x}_{1}$ in the interval $\left[0, \tilde{x}_{1}\right]$, because $\tilde{x}_{1}<\tilde{x}_{k}, \forall k>1$.

The maximum of intervals $\left[0, \tilde{x}_{1}\right],\left(\tilde{x}_{1}, x_{2}^{\infty}\right],\left(x_{2}^{\infty}, x_{3}^{\infty}\right], \ldots,\left(x_{k-1}^{\infty}, \tilde{x}_{k}\right]$ attain at the right of the intervals. The supremum of intervals $\left[\tilde{x}_{k}, x_{k+1}^{\infty}\right], \ldots,\left(x_{N}^{\infty}, k\right]$ attain at the left limitation of the intervals.

$$
\begin{aligned}
& W_{k}\left(\tilde{x}_{1}\right)-W_{k}\left(x_{j}^{\infty}\right) \\
& \quad=F\left(\tilde{x}_{1}\right)-p_{k} \tilde{x}_{1}-\left\{e^{-\tau_{k} \Sigma_{i=1}^{j-1} u_{i}^{m}}\left[F\left(x_{j}^{\infty}\right)-p_{k}\left(x_{j}^{\infty}-x_{j-1}^{\infty}\right)\right]-p_{k}\left(x_{j-1}^{\infty}-x_{j-2}^{\infty}\right) e^{\left.-\tau_{k} \Sigma_{1}^{j-2} u_{i}^{m}-\cdots-p_{k} \tilde{x}_{1}\right\}}\right.
\end{aligned}
$$




$$
\begin{aligned}
& =F\left(\tilde{x}_{1}\right)-e^{-\tau_{k} \sum_{i=1}^{j-1} u_{i}^{m}} F\left(x_{j}^{\infty}\right)+p_{k}\left(x_{j}^{\infty}-x_{j-1}^{\infty}\right) e^{-\tau_{k} \sum_{i=1}^{j-1} u_{i}^{m}}+p_{k}\left(x_{j-1}^{\infty}-x_{j-2}^{\infty}\right) e^{-\tau_{k} \Sigma_{1}^{j-2} u_{i}^{m}}+\cdots \\
& \quad+p_{k}\left(x_{2}^{\infty}-\tilde{x}_{1}\right) e^{-\tau_{k} u_{1}^{m}} \\
& >F\left(\tilde{x}_{1}\right)-e^{-\tau_{k} \sum_{i=1}^{j-1} u_{i}^{m}} F\left(x_{j}^{\infty}\right)
\end{aligned}
$$

$$
>0
$$

for $j \in\left\{\mathrm{i} \in \mathrm{N}^{+}: 2 \leq i \leq k-1\right\}$. Because of assumption (7a).

And $W_{k}\left(\tilde{x}_{1}\right)-W_{k}\left(\tilde{x}_{k}\right)$

$=F\left(\tilde{x}_{1}\right)-p_{k} \tilde{x}_{1}-\left\{e^{-\tau_{k} \sum_{i=1}^{k-1} u_{i}^{m}}\left[F\left(\tilde{x}_{k}\right)-p_{k}\left(\tilde{x}_{k}-x_{k-1}^{\infty}\right)\right]-p_{k}\left(x_{k-1}^{\infty}-x_{k-2}^{\infty}\right) e^{-\tau_{k} \sum_{1}^{k-2} u_{i}^{m}}-\cdots-p_{k} \tilde{x}_{1}\right\}$

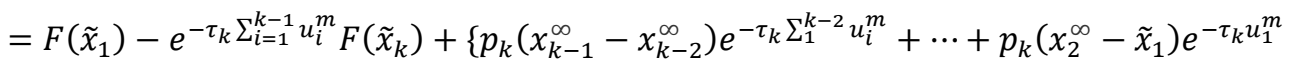

$>F\left(\tilde{x}_{1}\right)-e^{-\tau_{k} \sum_{i=1}^{k-1} u_{i}^{m} F\left(\tilde{x}_{k}\right)}$

$>0$

because of assumption (7b).

$W_{k}\left(\tilde{x}_{1}\right)-\lim _{x \rightarrow x_{j}^{\infty}} W_{k}(x)$

$=F\left(\tilde{x}_{1}\right)-p_{k} \tilde{x}_{1}$

$-\left\{e^{-\tau_{k} \sum_{i \in\left\{i \in \mathrm{N}^{+}: 1 \leq i \leq j, i \neq k\right\}} u_{i}^{m}} F\left(x_{j}^{\infty}\right)-p_{k}\left(x_{j}^{\infty}-x_{j-1}^{\infty}\right) e^{-\tau_{k} \sum_{i \in\left\{i \in \mathrm{N}^{+}: 1 \leq i \leq N-1, i \neq k\right\}} u_{i}^{m}} \cdots-p_{k} \tilde{x}_{1}\right\}$

$>F\left(\tilde{x}_{1}\right)-e^{-\tau_{k} \sum_{i \in\left\{i \in \mathrm{N}^{+}: 1 \leq i \leq j, i \neq k\right\}} u_{i}^{m}} F\left(x_{j}^{\infty}\right)$

$>0$

for $\forall j \in\left\{\mathrm{i} \in \mathrm{N}^{+}: k+1 \leq i \leq N\right\}$. Because of assumption (7c)

Therefore, $v_{i}^{*}(x, t), i \in\left\{\mathrm{i} \in \mathrm{N}^{+}: 1 \leq i \leq N\right\}$ yield a feedback Nash equilibrium.

Proof of Proposition 5.2

Suppose that agent $i, i \in\left\{\mathrm{i} \in \mathrm{N}^{+}: 2 \leq i \leq N\right\}$ use the strategies in (8).Then, we have

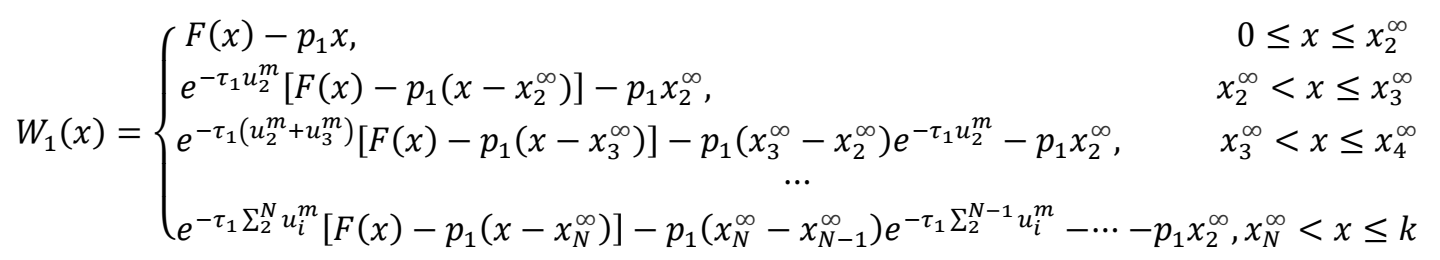

$F(x)-p_{1} x$ increase in the interval $\left[0, \tilde{x}_{1}\right]$, and decrease in the interval $\left[\tilde{x}_{1}, k\right]$.

We want to show that the maximum of $W_{1}(x)$ is attained at $x=x_{2}^{\infty}$.According to the assumption (9), we only need to show that

$$
\begin{gathered}
W_{1}\left(x_{2}^{\infty}\right) \geq W_{1}\left(x_{3}^{\infty}\right) \geq \cdots \geq W_{1}\left(x_{j}^{\infty}\right), W_{1}\left(x_{2}^{\infty}\right)>W_{1}\left(\tilde{x}_{1}\right) \\
W_{1}\left(x_{2}^{\infty}\right) \geq \lim _{x \rightarrow x_{j+1}^{\infty}}+W_{1}(x) \geq \lim _{x \rightarrow x_{j+2}^{\infty}}+W_{1}(x) \geq \cdots \geq \lim _{x \rightarrow x_{N}^{\infty+}} W_{1}(x) .
\end{gathered}
$$

and 


$$
\begin{aligned}
& W_{1}\left(x_{k}^{\infty}\right)-W_{1}\left(x_{k+1}^{\infty}\right) \\
& =e^{-\tau_{1} \sum_{2}^{k-1} u_{i}^{m}}\left[F\left(x_{k}^{\infty}\right)-p_{1}\left(x_{k}^{\infty}-x_{k-1}^{\infty}\right)\right]-p_{1}\left(x_{k-1}^{\infty}-x_{k-2}^{\infty}\right) e^{-\tau_{1} \sum_{2}^{k-2} u_{i}^{m}}-\cdots-p_{1} x_{2}^{\infty} \\
& -\left\{e^{-\tau_{1} \sum_{2}^{k} u_{i}^{m}}\left[F\left(x_{k+1}^{\infty}\right)-p_{1}\left(x_{k+1}^{\infty}-x_{k}^{\infty}\right)\right]-p_{1}\left(x_{k}^{\infty}-x_{k-1}^{\infty}\right) e^{-\tau_{1} \sum_{2}^{k-1} u_{i}^{m}}-\cdots-p_{1} x_{2}^{\infty}\right\}
\end{aligned}
$$

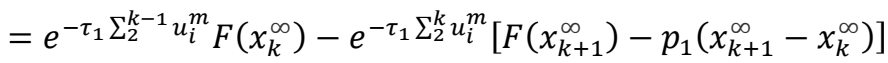

$$
\begin{aligned}
& >0
\end{aligned}
$$

Inequality is satisfied because of assumption (11). And $W_{1}\left(x_{2}^{\infty}\right)>W_{1}\left(\tilde{x}_{1}\right)$ is satisfied from assumption (12).

Now, we assume that the agent $i, i \in\left\{\mathrm{i} \in \mathrm{N}^{+}: 1 \leq i \leq N, i \neq k\right\}$ use $v_{i}^{*}(x, t)$ given by (8).Then,

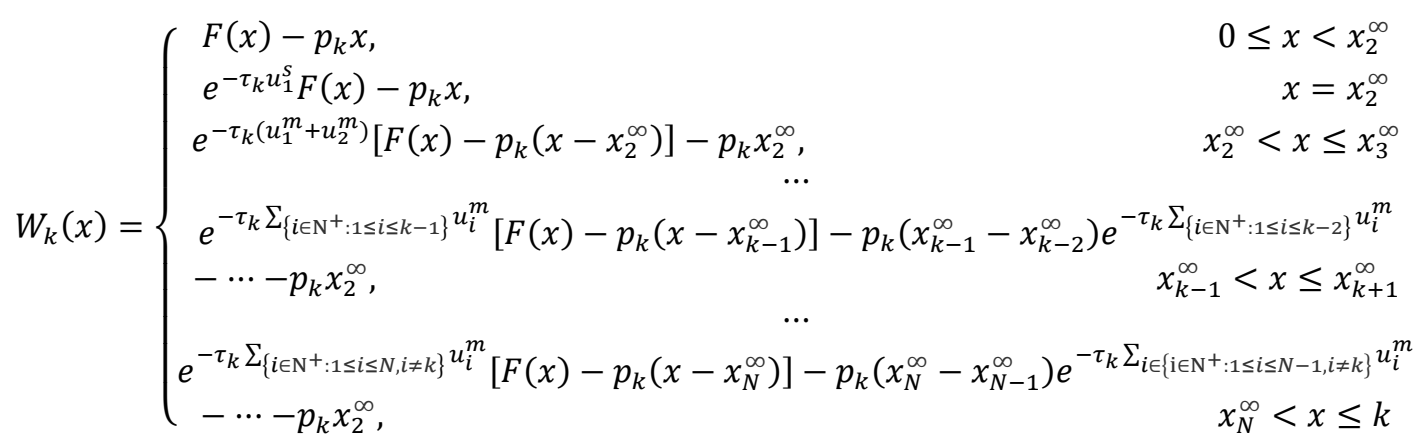

Case 1. $\tilde{x}_{k}<x_{2}^{\infty}$.Thus $F(x)-p_{k} x$ decreases in $\left[x_{2}^{\infty}, k\right]$.

$$
\begin{aligned}
& \lim _{x \rightarrow x_{j}^{\infty+}} W_{k}(x)-\lim _{x \rightarrow x_{j+1}^{\infty}}{ }^{+} W_{k}(x)=\left\{e^{-\tau_{k} \sum_{1}^{j} u_{i}^{m}} F\left(x_{j}^{\infty}\right)-p_{k}\left(x_{j}^{\infty}-x_{j-1}^{\infty}\right) e^{-\tau_{k} \sum_{1}^{j-1} u_{i}^{m}}-\cdots-p_{k} x_{2}^{\infty}\right\}
\end{aligned}
$$

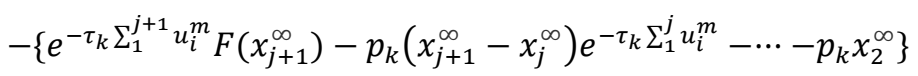

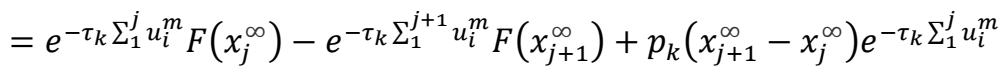

$$
\begin{aligned}
& >e^{-\tau_{k} \Sigma_{1}^{j} u_{i}^{m}} F\left(x_{j}^{\infty}\right)-e^{-\tau_{k} \Sigma_{1}^{j} u_{i}^{m}} F\left(x_{j+1}^{\infty}\right)+p_{k}\left(x_{j+1}^{\infty}-x_{j}^{\infty}\right) e^{-\tau_{k} \Sigma_{1}^{j} u_{i}^{m}} \\
& =e^{-\tau_{k} \sum_{1}^{j} u_{i}^{m}}\left\{\left[F\left(x_{j}^{\infty}\right)-p_{k} x_{j}^{\infty}\right]-\left[F\left(x_{j+1}^{\infty}\right)-p_{k} x_{j+1}^{\infty}\right]\right\} \\
& >0, j \in\left\{\mathrm{i} \in \mathrm{N}^{+}: 1 \leq i \leq N, i \neq k-1, i \neq k\right\} \\
& \lim _{x \rightarrow x_{k-1}^{\infty}+} W_{k}(x)-\lim _{x \rightarrow x_{k+1}^{\infty}+} W_{k}(x)>0 .
\end{aligned}
$$

And

$$
\begin{aligned}
W_{k}\left(x_{2}^{\infty}\right)-\lim _{x \rightarrow x_{2}^{\infty+}} W_{k}(x) & =e^{-\tau_{k} u_{1}^{s}} F\left(x_{2}^{\infty}\right)-p_{k} x_{2}^{\infty}-e^{-\tau_{k}\left(u_{1}^{m}+u_{2}^{m}\right)} F\left(x_{2}^{\infty}\right)+p_{k} x_{2}^{\infty} \\
& =\left[e^{-\tau_{k} u_{1}^{s}}-e^{-\tau_{k}\left(u_{1}^{m}+u_{2}^{m}\right)}\right] F\left(x_{2}^{\infty}\right)>0 .
\end{aligned}
$$

Hence, the maximum of $W_{k}(x)$,subject to $x \geq x_{2}^{\infty}$, is attained at $x=x_{2}^{\infty}$.

Case 2. $\tilde{x}_{k} \geq x_{2}^{\infty}$. Thus $F(x)-p_{k} x$ increase in $\left[0, \tilde{x}_{k}\right]$ and decreases in $\left[\tilde{x}_{k}, k\right]$.Suppose that $\tilde{x}_{k}$ satisfies that $x_{m}^{\infty}<\tilde{x}_{k}<x_{m+1}^{\infty}, \forall m \in\left\{\mathrm{i} \in \mathrm{N}^{+}: 2 \leq i \leq N-1\right\}$, we have proofed that 


$$
\lim _{x \rightarrow x_{j}^{\infty+}} W_{k}(x)-\lim _{x \rightarrow x_{j+1}^{\infty}} W_{k}(x)>0, \text { if } j \geq m+1 .
$$

We only need to proof that $W_{k}\left(x_{2}^{\infty}\right)>W_{k}\left(\tilde{x}_{k}\right)$ and $W_{k}\left(x_{2}^{\infty}\right) \geq W_{k}\left(x_{3}^{\infty}\right) \geq \cdots \geq W_{k}\left(x_{m}^{\infty}\right)$.

$$
\begin{gathered}
W_{k}\left(x_{j}^{\infty}\right)-W_{k}\left(x_{j+1}^{\infty}\right)=\left\{e^{-\tau_{k} \sum_{\left\{i \in \mathrm{N}^{+}: 1 \leq i \leq j-1, i \neq k\right\}} u_{i}^{m}}\left[F\left(x_{j}^{\infty}\right)-p_{k}\left(x_{j}^{\infty}-x_{j-1}^{\infty}\right)\right]\right. \\
\left.-p_{k}\left(x_{j-1}^{\infty}-x_{j-2}^{\infty}\right) e^{-\tau_{k} \sum_{\left\{i \in \mathrm{N}^{+}: 1 \leq i \leq j-2, i \neq k\right\}} u_{i}^{m}}-\cdots-p_{k} x_{2}^{\infty}\right\} \\
-\left\{e^{-\tau_{k} \sum_{\left\{i \in \mathrm{N}^{+}: 1 \leq i \leq j, i \neq k\right\}} u_{i}^{m}}\left[F\left(x_{j+1}^{\infty}\right)-p_{k}\left(x_{j+1}^{\infty}-x_{j}^{\infty}\right)\right]\right. \\
\left.\quad-p_{k}\left(x_{j}^{\infty}-x_{j-1}^{\infty}\right) e^{-\tau_{k} \sum_{\left\{i \in \mathrm{N}^{+}: 1 \leq i \leq j-1, i \neq k\right\}} u_{i}^{m}}-\cdots-p_{k} x_{2}^{\infty}\right\} \\
=e^{-\tau_{k} \sum_{\left\{i \in \mathrm{N}^{+}: 1 \leq i \leq j-1, i \neq k\right\}} u_{i}^{m}} F\left(x_{j}^{\infty}\right)-\left\{e^{-\tau_{k} \sum_{\left\{i \in \mathrm{N}^{+}: 1 \leq i \leq j, i \neq k\right\}} u_{i}^{m}}\left[F\left(x_{j+1}^{\infty}\right)-p_{k}\left(x_{j}^{\infty}-x_{j-1}^{\infty}\right)\right]\right\} \\
\geq 0, j \in\left\{\mathrm{i} \in \mathrm{N}^{+}: 2 \leq i \leq m-1, i \neq k-1, i \neq k\right\} .
\end{gathered}
$$

Inequality satisfies because assumption (13). Hence we have proofed that $W_{k}\left(x_{2}^{\infty}\right) \geq W_{k}\left(x_{3}^{\infty}\right) \geq \cdots \geq W_{k}\left(x_{m}^{\infty}\right)$ and at most one inequality satisfies with equality.

$$
\begin{aligned}
& W_{k}\left(x_{2}^{\infty}\right)-W_{k}\left(\tilde{x}_{k}\right) \\
= & {\left[e^{-\tau_{k} u_{1}^{s}} F\left(x_{2}^{\infty}\right)-p_{k} x_{2}^{\infty}\right]-\left\{e^{-\tau_{k} \sum_{\left\{i \in \mathrm{N}^{+}: 1 \leq i \leq m, i \neq k\right\}} u_{i}^{m}}\left[F\left(\tilde{x}_{k}\right)-p_{k}\left(\tilde{x}_{k}-x_{m}^{\infty}\right)\right]\right.} \\
& \left.-p_{k}\left(x_{m}^{\infty}-x_{m-1}^{\infty}\right) e^{-\tau_{k} \sum_{i \in\left\{i \in \mathrm{N}^{+}: 1 \leq i \leq N-1, i \neq k\right\}} u_{i}^{m}}-\cdots-p_{k} x_{2}^{\infty}\right\}>0
\end{aligned}
$$

because of assumption (14).

Hence, the maximum of $W_{k}(x)$, subject to $x \geq x_{2}^{\infty}$, is attained at $x=x_{2}^{\infty}$.

To complete the proof, assumption (10) guarantees that strategy $u_{1}^{s}$ is feasible.

Proof of Proposition 5.3

In the process of proofing Proposition 5.1., we obtain

$$
W_{k}(x)=F(x) e^{-\sum_{i \neq k} \tau_{i} u_{i}(x)}-p_{k} \int_{0}^{x} e^{-\sum_{i \neq k} \tau_{k} u_{i}(z)} d z, \forall x \in[0, \mathrm{k}], k \in\left\{\mathrm{i} \in \mathrm{N}^{+}: 1 \leq i \leq N\right\}
$$

If everyone uses the strategies above, then

$$
W_{k}(x)=\left[F(x)-p_{k} x\right] e^{-\sum_{i \neq k} \tau_{k} u_{i}^{m}}
$$

Under assumption (17), $\frac{d\left(F(x)-p_{i} x\right)}{x}=r+b-p_{i}-\frac{2 x r}{k}<0$. Therefore, the maximum of $W_{k}(x)$ is attained at $x=0$. The optimal strategies corresponding to most rapidly approaching path is given above. The playability of strategies (15) originates from the fact that $\dot{x}=0$ for $x=0$, irrelevant to the value $u_{i}^{0}$. 


\section{Proof of Proposition 5.4}

From (7), we can see that $W_{i}(x)$ is constant on some interval if and only if it satisfies that

$$
W_{k}^{\prime \prime}(x)=e^{-\sum_{i \neq k} \tau_{k} u_{i}(x)}\left[-\tau_{k} \sum_{i \neq k} v^{\prime}{ }_{i}(x) F(x)+F^{\prime}(x)-p_{k}\right]=0
$$

It is equivalent to

$$
\sum_{i \neq k} v_{i}^{\prime}(x)=\frac{F^{\prime}(x)-p_{k}}{\tau_{k} F(x)}
$$

Through integration, we get

$$
\sum_{i \neq k} v_{i}(x)=G_{k}(x)+\text { constant. } k \in\left\{\mathrm{i} \in \mathrm{N}^{+}: 1 \leq i \leq N\right\} .
$$

Equal to

$$
v_{k}(x)=\frac{\sum_{1}^{N} G_{i}(x)}{N-1}-G_{k}(x)+\text { constant }
$$

Therefore, $W_{k}(x), k \in\left\{\mathrm{i} \in \mathrm{N}^{+}: 1 \leq i \leq N\right\}$ is unchangeable on the interval $[\underline{x}, \bar{x})$. In addition, (19) implies that

$$
W_{k}(x)=F(x)-p_{k}, \text { for } x \leq \underline{x},
$$

and $W_{k}(x)$ is continuous at $x=\underline{x}$. Condition (18b) guarantees that $v_{k}(x) \in\left[0, u_{j}^{m}\right]$ for $x \in[\underline{x}, \bar{x}]$ because

$$
\begin{aligned}
{\left[\frac{\sum_{1}^{N} G_{i}(x)}{N-1}-G_{k}(x)\right]-\left[\frac{\sum_{1}^{N} G_{i}(\underline{x})}{N-1}-G_{k}(\underline{x})\right] } & <\left[\frac{\sum_{1}^{N} G_{i}(x)}{N-1}-G_{k}(x)\right] \\
& <\left[\frac{\sum_{1}^{N} G_{i}(\bar{x})}{N-1}-G_{k}(\underline{x})\right] \\
& <u_{j}^{m} .
\end{aligned}
$$

Therefore, the optimal response of agent $k$ given strategies $v_{i}, i \in\left\{\mathrm{i} \in \mathrm{N}^{+}: 1 \leq i \leq N, i \neq k\right\}$ is a most rapidly approach path to the interval $[\underline{x}, \bar{x})$, and staying in this interval. It is feasible, because

$\left.\dot{x}\right|_{x=\underline{x}}=F(\underline{x})-b \underline{x}>0$,

$\left.\dot{x}\right|_{x=\bar{x}}=F(x)-b x e^{\sum_{i=1}^{N} \bar{u}_{i}} \leq F(x)-b x e^{\sum_{i=1}^{N}\left[\frac{\sum_{1}^{N} G_{i}(\bar{x})}{N-1}-G_{k}(\underline{x})\right]} \leq F(x)-b x e^{u^{s}}=0$.

The last inequality comes from (23c). And the playability of the strategy $v_{k}(x)$ in (19) is guaranteed by the assumption.

\section{Proof of Proposition 6.1}

Proof. Define the value functions $V^{k}=\max _{u_{i}} \int_{t}^{T} e^{-p_{k} s} c_{k} x e^{u_{k}+a_{k} \sum_{i \neq k} u_{i}} d s+e^{-p_{k} T} S_{i} x(T)$, such that

$$
V^{k}(T, x)=e^{-p_{k} T} S_{k} x
$$

If there is an optimal strategy combination, through Hamilton-Jacobi-Bellman equations we must have: 


$$
-V_{t}^{k}=\max _{u_{i}}\left\{e^{-p_{k} t} c_{k} x e^{u_{k}+a_{k} \sum_{i \neq k} u_{i}}+V_{x}^{k}\left[r x\left(1-\frac{x}{k}\right)-b x\left(e^{\sum_{i=1}^{N} u_{i}}-1\right)\right]\right\} .
$$

The strategy combination $\left\{v_{i}^{*}(t, x)\right\}$, where

$$
v_{i}^{*}(t, x)=v_{i}\left[t, x, V_{x}^{1}(t, x), \cdots, V_{x}^{N}(t, x)\right],
$$

is obtained by maximizing in (A4), satisfies the Nash condition in Definition 3.5, and is a feedback Nash equilibrium if we find functions $V^{k}(T, x)$ that can solve Equation (A4) and satisfies (A3).

We construct the optimal strategies in a region of the playing space where $V^{i}$ is $C^{1}, x \in[0, k]$,and $u_{i}$ satisfies $0 \leq u_{i} \leq u^{m}$. Maximizing (A4) pointwise needs

$$
e^{-p_{k}} c_{k} x e^{u_{k}+a_{k} \sum_{i \neq k} u_{i}}-V_{x}^{k} b x e^{\sum_{i=1}^{N} u_{i}}=0
$$

which implies that

$$
\sum_{i \neq k} u_{i}=\frac{1}{1-a_{k}} \ln \frac{e^{-p_{k} t} c_{k}}{V_{x}^{k} b}, k \in\left\{\mathrm{i} \in \mathrm{N}^{+}: 1 \leq i \leq N\right\}
$$

and substitute it into (A4) we get

$$
V_{t}^{k}+V_{x}^{k}\left[r x\left(1-\frac{x}{k}\right)+b x\right]=0
$$

A solution of (A6) can be written as

$$
V^{k}(t, x)=\frac{e^{-p_{k} T} S_{k}(r+b)}{\frac{r}{k}+\left(\frac{r+b}{x}-\frac{r}{k}\right) e^{(r+b)(t-T)}} .
$$

Take derivative of (A7) to $x$ we get

$$
V_{x}^{k}(t, x)=\frac{e^{-p_{k} T} S_{k} K^{2} e^{(r+b)(t-T)}}{\left[K f(t)+x\left[1-e^{(r+b)(t-T)}\right]\right]^{2}},
$$

where $K=\frac{k(r+b)}{r}$.

It shows that $V_{x}^{k}(t, x)$ is positive for all $(t, x)$. Because $V^{k}$ does not depend on $x_{0}$, we can interpret it as player k's value function, and $V^{k}$ represents the player $k^{\prime}$ optimal payoff in subgames starting at any time $\mathrm{t}$ with initial state of value $x(t)$.

Solve the system of equations (A5), we get

$$
u_{k}=\frac{\sum_{i \neq k}\left(\frac{1}{1-a_{i}} \ln \frac{e^{-p_{i} t} c_{i}}{V_{x}^{i} b}\right)}{N-1}-\frac{1}{1-a_{k}} \ln \frac{e^{-p_{k}} c_{k}}{V_{x}^{k} b},
$$

where $k \in\left\{\mathrm{i} \in \mathrm{N}^{+}: 1 \leq i \leq N\right\}$.

For $u_{k} \in\left[0, u_{k}^{m}\right], u_{k}$ can only be zero. And

$$
\frac{1}{1-a_{i}} \ln \frac{e^{-p_{i} t} c_{i}}{V_{x}^{i} b}=\frac{1}{1-a_{j}} \ln \frac{e^{-p_{j} t} c_{j}}{V_{x}^{j} b}=\delta, \forall i, j \in\left\{\mathrm{i} \in \mathrm{N}^{+}: 1 \leq i \leq N\right\} .
$$

where $\delta$ is a constant.

Substitute (A8) into (A10), we get 


$$
\frac{1}{1-a_{i}}\left[\ln \frac{c_{i}\left[K e^{(r+b)(t-T)}+x\left[1-e^{(r+b)(t-T)}\right]\right]^{2}}{b S_{i} K^{2} e^{(r+b)(t-T)}}+p_{i}(T-t)\right]=\delta
$$

Through this equation we can see that if $a_{i}, p_{i}, c_{i}, r, b$ increase $S_{i}, k$ must increase as well to make the equation still hold.

Substitute (A8) into (A9), we get

$$
\begin{gathered}
u_{k}=\frac{\sum_{i \neq k} \frac{1}{1-a_{i}}\left[\ln \frac{c_{i}\left[K e^{(r+b)(t-T)}+x\left(1-e^{(r+b)(t-T)}\right)\right]^{2}}{b S_{i} K^{2} e^{(r+b)(t-T)}}+p_{i}(T-t)\right]}{N-1} \\
-\frac{1}{1-a_{k}}\left[\ln \frac{c_{k}\left[K e^{(r+b)(t-T)}+x\left(1-e^{(r+b)(t-T)}\right)\right]^{2}}{b S_{k} K^{2} e^{(r+b)(t-T)}}+p_{k}(T-t)\right],
\end{gathered}
$$

Take partial derivative of $u_{k}$ to $a_{k}, c_{k}, S_{k}, p_{k}$ we get

$$
\frac{\partial u_{k}}{\partial a_{k}}<0, \frac{\partial u_{k}}{\partial c_{k}}<0, \frac{\partial u_{k}}{\partial S_{k}}>0, \frac{\partial u_{k}}{\partial p_{k}}<0 .
$$

\section{Copyrights}

Copyright for this article is retained by the author(s), with first publication rights granted to the journal.

This is an open-access article distributed under the terms and conditions of the Creative Commons Attribution license (http://creativecommons.org/licenses/by/4.0/). 